\title{
POBREZA RURAL E DESIGUALDADE DE RENDA NA AMAZÔNIA: UM ESTUDO DA PRODUÇÃO FAMILIAR RURAL DO ESTADO DO ACRE
}

\section{RURAL POVERTY AND INCOME INEQUALITY IN THE AMAZON: A STUDY OF RURAL FAMILY PRODUCTION IN THE STATE OF ACRE}

\author{
Raimundo Cláudio Gomes Maciel ${ }^{1}$ \\ Pedro Gilberto Cavalcante Filho ${ }^{2}$ \\ Tiago de Oliveira Loiola ${ }^{3}$ \\ Geso Willian da Silva Marçal ${ }^{4}$ \\ Dieime Lopes de Souza ${ }^{5}$
} Resumo: No Brasil, a pobreza e a insegurança alimentar concentrada no meio rural tem chamado bastante atenção por sua amplitude, principalmente no âmbito da agricultura familiar, onde a produção para o autoconsumo está no centro da reprodução familiar. Dessa forma, o presente trabalho objetiva identificar a relação entre a segurança alimentar, o autoconsumo e a pobreza da produção familiar rural do Estado do Acre. Especificamente, busca-se verificar os indicadores socioeconômicos, bem como os níveis de autoconsumo da produção familiar rural acreana. Para tanto, são utilizados indicadores econômicos adequados e específicos para estes estudos a partir dos estratos sociais identificados pela linha de pobreza no Estado. Os resultados demonstram um crescente número de famílias nas linhas de pobreza e extrema pobreza, elevando o número de pessoas em situação de insegurança alimentar, ocasionados, sobretudo pelo baixo autoconsumo.

Palavras-chave: Autoconsumo, pobreza rural, agricultura familiar.

Abstract: In Brazil, poverty and food insecurity concentrated in rural areas have attracted a lot of attention because of their breadth, especially in the context of family farming, where production for self-consumption is at the heart of family reproduction. Thus, the present work aims to identify the relationship between food security, self-consumption and poverty of rural family production in the State of Acre. Specifically, it is sought to verify the socioeconomic indicators, as well as the levels of self-consumption of the rural family production in Acre. To do so, appropriate and specific economic indicators are used for these studies from the social strata identified by the poverty line in the State. The results show an increasing number of families in the poverty and extreme poverty lines, increasing the number of people in food insecure situations, caused mainly by low self-consumption.

Key words: Self-consumption, rural poverty, family farming.

\footnotetext{
1 Economista, Doutor em Economia pelo Instituto de Economia da Universidade Estadual de Campinas (IE/UNICAMP). Professor da Universidade Federal do Acre (UFAC). E-mail: rcgmaciel@ufac.br

2 Economista, Mestrando em Desenvolvimento Econômico pelo Instituto de Economia da Universidade Estadual de Campinas (IE/UNICAMP). E-mail: pedro.gilberto@hotmail.com

3 Economista, Mestre em Desenvolvimento Regional pela Universidade Federal do Acre (UFAC). Professor da Universidade Federal de Rondônia. E-mail: tiago.loiola@yahoo.com.br

${ }^{4}$ Tecnólogo Financeiro. E-mail: gesofrauner@hotmail.com

${ }^{5}$ Historiadora pela Universidade Federal do Acre (UFAC). E-mail: dieimelopes@hotmail.com.
} 


\section{INTRODUÇÃO}

A agricultura familiar passou por diversas transformações estruturais e conceituais ao longo do tempo, mostrando que a diferença entre o camponês e o agricultor familiar ocorria pelo modelo de produção. Enquanto o camponês tinha uma produção "tradicional", o agricultor familiar conhece a melhor forma de utilizar seus meios de produção, até mesmo adaptar-se a uma exigência do mercado (CHAYANOV, 1974; WANDERLEY, 2003).

No decorrer dos anos a agricultura familiar recebeu diversos rótulos, iniciando no camponês, passando por pequeno produtor, agricultor de subsistência, e chegando, então, em agricultor familiar. Entretanto, percebe-se que mesmo ocorrendo essas mudanças na nomenclatura, o sujeito sempre foi o mesmo, porém, com diferentes estilos de produção. Essas mudanças sempre vinham com choques estruturais, com a finalidade de incentivar a produção rural (WANDERLEY, 1996; OLALDE E PORTUGAL, 2004).

As grandes diferenças da dinâmica produtiva entre as regiões brasileiras refletem-se dentro da agricultura familiar, uma vez que nas regiões mais desenvolvidas a produção agrícola familiar está inserida no mercado, porém, em regiões subdesenvolvidas há uma dificuldade produtiva até para 0 autoconsumo (TURPIN, 2009). Assim, estudos apontam que esse setor necessita de políticas adequadas para cada região de forma que auxilie 0 desenvolvimento das atividades produtivas dos agricultores mediante a oferta de condições básicas, como a infraestrutura adequada, acesso à tecnologia, acesso a mercados dinâmicos, disponibilidade de crédito adequado aos padrões desse público, permitindo a sua reprodução social no meio rural (GUANZIROLI et. al, 2001; NAVARRO, 2001; SOUZA FILHO, et. al, 2011).

A reprodução social é definida como a relação entre os indivíduos com sua família e a interação com o ambiente social que estão inseridos. Para tanto, a reprodução social da agricultura familiar ocorre a partir das dimensões de ciclo curto, que está relacionado aos aspectos econômicos, como trabalho, conhecimento tradicional e recursos naturais para suprir a demanda familiar; e de ciclo longo, que são as características familiares naturais de reprodução e continuidade no campo, sendo que este último tem enfrentado um grande problema devido as culturas e conhecimentos tradicionais anteriormente passadas de geração em geração não ocorrem mais (SCHNEIDER, 2003; SPANEVELLO, LAGO, 2010; PELEGRINI, GAZOLLA, 2009; MATTE, SPANEVELLO, AZEVEDO, 2010).

Por outro lado, um dos grandes gargalos encontrados no meio rural que dificultam a reprodução social das famílias no campo é a desigualdade de renda, que se explica como herança de um processo de injustiça social do resultado da exploração da mão de obra perpetuado por séculos. Destarte, observou-se o aumento do número de trabalhadores assalariados em detrimento do número de trabalhadores sem remuneração, agravando ainda mais as desigualdades sociais no campo (BARROS et. al, 2001; CACCIAMALI, 2002).

No entanto, na última década, o Governo Federal, através da implantação de programas sociais de distribuição de renda, apontou redução 
significativa nos indicadores de pobreza (ROCHA, 2006). Hoffman (2009, p. 2) afirma que, "a desigualdade da distribuição de renda no Brasil sofreu substancial redução nos últimos anos, mas continua sendo muito elevada em comparação com outros países".

A pobreza é caracterizada como a insuficiência de recursos para assegurar as condições básicas de subsistência e de bem-estar e está dividida em duas linhas: unidimensional e multidimensional. A primeira, se restringe ao dimensionamento apenas através da renda auferida, no qual o Banco Mundial tomava como referência 1 dólar ao dia até o início dos anos 2000. A segunda amplia a forma de mensurar a pobreza, uma vez que além da renda monetária são levados em consideração os aspectos sociais, como a ausência de educação, saúde, infraestrutura etc. (SILVA, 2009; OLIVEIRA, 2010).

Recentemente, o Banco Mundial adotou uma nova métrica para determinar a quantidade de pessoas que vivem abaixo da linha de pobreza, considerando a realidade de cada país. Para países de renda média baixa, considera-se pessoas em situação de pobreza as que recebem o valor de até US\$ 3,20 por dia. Países considerados de renda média alta - entre os quais o Brasil está incluído - a linha de pobreza determinada é de US $\$ 5,50$ por dia. $O$ Programa das Nações Unidas para o Desenvolvimento (PNUD), instituição subordinada a Organização das Nações Unidas (ONU), estabeleceu como linha limítrofe para considerar que uma pessoa está em situação de extrema pobreza o valor de US\$1,25 (WORLD BANK, 2016).

Portanto, não é coerente calcular a pobreza rural apenas pela linha unidimensional, pois no meio rural a família produz alimentos que não vão para o mercado, tornando um valor não monetário, utilizando o alimento apenas para o autoconsumo da família. Assim, o cálculo da pobreza rural seria superficial, desconsiderando a qualidade de vida das famílias no meio rural (NEDER, 2008).

Nesse aspecto, a produção de autoconsumo sofre algumas críticas e é vista como um retrocesso, uma vez que o princípio dessa produção era somente suprir as necessidades das famílias, sem buscar lucro com a produção, tornando-se uma atividade antieconômica. No entanto, esse tipo de produção é uma das fortalezas da agricultura familiar e fundamental para o desenvolvimento desse setor no campo, de forma que minimize a sua dependência do mercado pelo menos no tocante à demanda de alimentos (WOLF, 1970; DOMBEK, 2006; SCHNEIDER, GAZOLLA, 2007).

Entretanto, segundo Wolf (1970), à medida que a sociedade vai se desenvolvendo, o produtor familiar rural deixa de produzir o necessário e tende a produzir somente para obtenção de lucro, introduzindo novas técnicas e criações, prejudicando a produção para o autoconsumo e despertando preocupação a respeito da segurança alimentar.

O surgimento da expressão "Segurança Alimentar" ocorreu no período da Primeira Guerra Mundial. Devido à existência de uma grande quantidade de pessoas vivendo na vulnerabilidade em consequência da escassez de alimentos. Fato que se agravou pelo o predomínio entre os países ricos sobre os países pobres, fazendo com que houvesse uma dependência muito grande de alimentos por parte dos países pobres (MALUF, 2007; DEVES E FILIPPI, 2008). 
A insegurança alimentar no meio rural brasileiro é um fato marcado pela pobreza, Hoffman (1995) destaca que o problema da fome no Brasil não ocorre a pouca disponibilidade global de alimentos, mas à pobreza de grande parte da população.

A questão central desse trabalho é: qual o papel do autoconsumo da produção familiar rural do Estado do Acre no que diz respeito a distribuição de renda e garantia de segurança alimentar?

O presente trabalho tem como objetivo verificar a relação entre a pobreza, segurança alimentar e o autoconsumo da produção familiar rural do Estado do Acre. Especificamente, busca-se identificar os principais indicadores socioeconômicos da produção familiar rural no Estado, bem como os níveis de autoconsumo e de distribuição de renda no meio rural acreano a partir do nível de vida.

A hipótese adotada para nortear este trabalho é que o autoconsumo das famílias produtoras rurais acreanas garantem a situação de segurança alimentar e apresentam distribuição de renda adequada.

Os procedimentos metodológicos e o banco de dados utilizados no presente trabalho são do Projeto de Análise Socioeconômica dos Sistemas Básicos de Produção Familiar Rural do Estado do Acre (ASPF), desenvolvido pelo Centro de Ciências Jurídicas e Sociais Aplicadas (CCJSA) da Universidade Federal do Acre (UFAC).

A importância deste trabalho se justifica pela ausência de estudos relacionados a esta temática no Estado do Acre, bem como pela necessidade de monitoramento e avaliação efetiva que possam auxiliar no desenvolvimento e consolidação da agricultura familiar, em particular nas zonas rurais do Estado do Acre, buscando subsidiar políticas públicas voltadas para o setor agrícola, notadamente em relação às questões de segurança alimentar e autoconsumo.

\section{METODOLOGIA}

O Estado do Acre é uma das 27 unidades federativas do Brasil, que possui 733.559 mil habitantes, dos quais 201.280 mil residem na zona rural do estado, ou seja, a população rural acreana corresponde a 27\% (IBGE, 2011).

Este estudo tem como objeto a produção familiar rural do estado do Acre, sendo a análise voltada para o autoconsumo, a segurança alimentar e a distribuição de renda das famílias rurais nas mesorregiões do Vale do Acre e do Vale do Juruá.

$\mathrm{Na}$ mesorregião do Vale do Acre foram realizados dois levantamentos em um intervalo de quase dez anos, sendo o primeiro período o ano agrícola 1996/1997 e o segundo 2005/2006. Já na mesorregião do Vale do Juruá considerou-se os períodos 1999/2000 e 2006/2007. Assim, as pesquisas realizadas permitiram um estudo aprofundado e comparativo entre as duas mesorregiões do Estado do Acre, além de identificar as principais transformações produtivas, econômicas e sociais ocorridas na produção familiar rural do Estado do Acre em um intervalo de dez anos. 
Mapa 1 - Localização da área e estudo e a Divisão Política do estado do Acre.

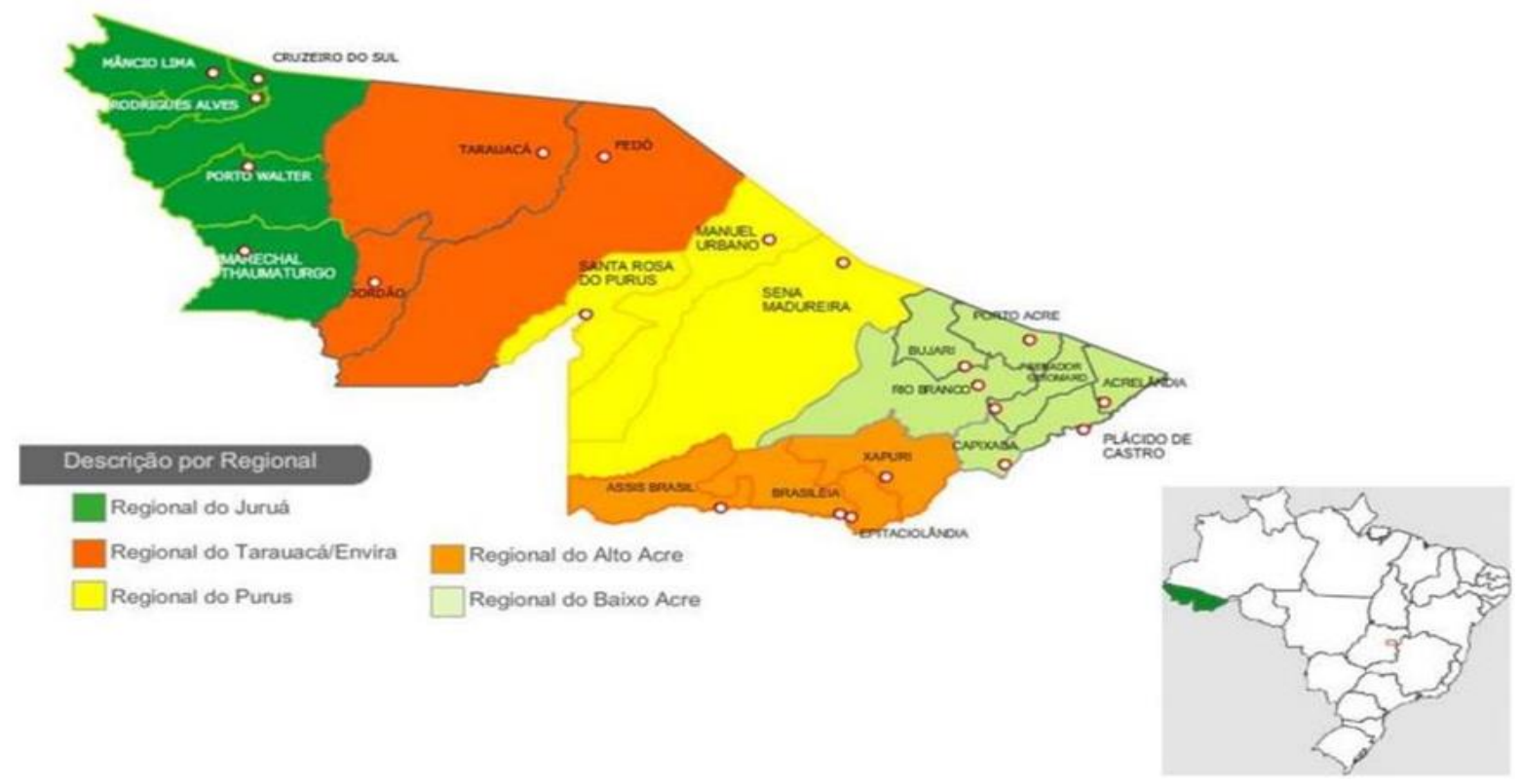

Fonte: ACRE (2011).

Este trabalho tem informações dos resultados obtidos da pesquisa de campo, no referido assentamento pelo projeto de pesquisa "Análise Socioeconômica de Sistemas de Produção Familiar Rural no estado do Acre", $\left(\mathrm{ASPF}^{6}\right)$, desenvolvido pelo Centro de Ciências Jurídicas e Sociais Aplicadas (CCJSA), da Universidade Federal do Acre (UFAC).

No projeto ASPF foram construídos vários indicadores para a avaliação econômica da produção familiar rural no Acre, que vão desde os tradicionais até os que somente se aplicam à produção familiar rural. Os principais indicadores econômicos utilizados neste trabalho estão sucintamente descritos a seguir:

Autoconsumo $(A C)$

$$
\mathbf{A C}=\sum_{\mathbf{v}=\mathbf{1}}^{\mathbf{n}}(\mathbf{Q b c p}) \mathbf{v} \cdot \mathbf{p v}
$$

Significando:

$\mathbf{A C}=$ autoconsumo

(Qbcp)v = quantidade do bem de autoconsumo produzido v

$\mathbf{p v}=$ preço unitário do bem de autoconsumo produzido $\mathbf{v}$

$\mathbf{v}=$ itens de bens de autoconsumo produzidos $(v=1,2, \ldots, n)$

Resultado Bruto (RB)

O resultado bruto é basicamente o valor da produção destinada ao mercado, obtido pela seguinte fórmula:

Sendo:

$$
R B=Q m . p p
$$

\footnotetext{
${ }^{6} \mathrm{O}$ projeto ASPF desenvolve pesquisas socioeconômicas na área da produção familiar rural na região acreana desde 1996, com diversas publicações sobre o tema. Para mais informações ver:

http://aspf.wordpress.com/
} 


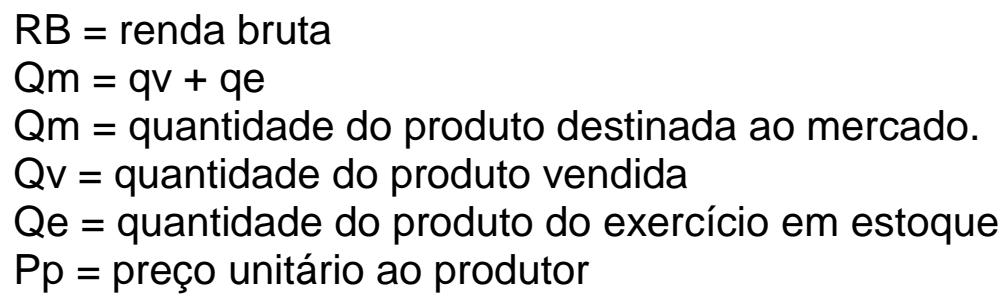

Margem Bruta Familiar (MBF)

Margem bruta familiar (MBF) é o resultado líquido específico e próprio para indicar o valor monetário disponível para a subsistência da família, inclusive uma eventual elevação do nível de vida, se o montante for suficiente.

Sendo:

$$
\text { MBF }=\text { RB }-(C V-C f t f)
$$

$\mathbf{R B}=$ renda bruta

CV $=$ custos variáveis

Cftf = custo real da força de trabalho familiar

Utiliza-se o valor do MBF/Qh/d para comparar com o custo de oportunidade, o qual nesse trabalho trata-se do valor de uma diária de trabalho paga na região.

MBF/Qh/d - índice de remuneração da mão de obra familiar;

$\mathrm{d}=$ quantidade de dias de trabalho.

Nível de Vida (NV)

O Nível de Vida (NV) é a totalidade do valor apropriado pelo produtor familiar, inclusive valores imputados, deduzidas as obrigações financeiras com empréstimos. O nível de vida é calculado por:

$$
\mathbf{N V}=(\mathbf{M B F}+\mathbf{A C}+\mathbf{C j i c c})-\mathbf{A A}
$$

Sendo:

MBF = Margem Bruta Familiar

$\mathbf{A C}=$ Autoconsumo

Cjicc = juros imputados ao capital circulante.

AA = amortizações anuais de empréstimos

Valor dos Bens de Consumo Comprado no Mercado (Vbcc)

O Valor dos Bens de Consumo Comprados no Mercado (VBCC) é um indicador que mede a quantidade em valores em reais comprados pelo agricultor família, a fórmula é dada por:

$$
\text { Vbcc }=\sum_{\mu=1}^{n}(\text { Qbcc }) \mu \cdot p \mu
$$

Sendo:

Vbcc $=$ valor dos bens e serviços de consumo comprados

Qbcc = quantidade de bens e serviços de consumo comprados $u$

pu = preço unitário de um bem e/ou serviço de consumo comprado

$\mathbf{u}=$ itens de bens e serviços de consumo $(u=1,2, \ldots, n)$

Linha de Dependência do Mercado

Definem-se como linha de dependência do mercado os valores medianos gastos com bens e serviços de consumo no mercado, adicionados 
das compras relacionadas à reposição do capital fixo (máquinas, equipamentos, ferramentas, benfeitorias, etc.) disponível para a manutenção dos meios de produção existentes.

Índice de Eficiência Econômica (IEE)

É o indicador de benefício/custo. É definido pela seguinte fórmula:

$$
I E E=\frac{R B}{C T}
$$

Sendo:

RB = Renda Bruta;

$\mathrm{CT}=$ Custos Totais.

- IEE > 1, a situação é de lucro.

- $\quad \mathrm{IEE}<1$, a situação é de prejuízo.

- $\quad \mathrm{IEE}=1$, a situação é de equilíbrio.

Índice de Gini

O Índice de Gini é uma medida de concentração ou desigualdade utilizada na análise da distribuição de renda e pode ser utilizado para medir o grau de concentração de qualquer distribuição estatística.

Segurança Alimentar

Utiliza-se o indicador da Pesquisa Nacional por Amostra de Domicílio (PNAD) como método para avaliar o nível de segurança alimentar e insegurança alimentar dos produtores familiares rurais do Estado do Acre. De acordo com o procedimento a PNAD 2004, as famílias que vivem com renda per capita/mês abaixo de 1/4 salário mínimo ${ }^{7}$ estão em situação de insegurança alimentar.

Embora em algumas instituições internacionais o indicador para medir a extrema pobreza seja US\$ 1 ao dia, no Brasil é usada uma metodologia diferente, conforme o Relatório Nacional de Acompanhamento dos Objetivos do Desenvolvimento do Milênio, a extrema pobreza no Brasil é utilizada como parâmetro de $1 / 4$ salário mínimo, ou seja, o valor de um quarto do salário mínimo de renda per capita por mês para dimensionar a extrema pobreza e meio salário mínimo de renda per capita por mês para dimensionar a pobreza.

Os dados analisados referentes à segurança alimentar foram coletados por meio de questionários sobre o desempenho econômico das famílias rurais do Estado do Acre, em particular o Nível de Vida (NV), que servirá para calcular o nível de segurança alimentar e insegurança alimentar.

Tabela 1 - Classificação da faixa de renda, valores confirme o salário mínimo.

\begin{tabular}{cc}
\hline Classe & Faixas de Renda (SM) \\
\hline B & $\mathrm{NV}>4 \mathrm{SM} / \mathrm{mês} ;$ \\
$\mathrm{C}$ & $2 \mathrm{SM} / \mathrm{mês}<\mathrm{NV}<4 \mathrm{SM} / \mathrm{mês} ;$ \\
$\mathrm{D}-\mathrm{Pobreza}$ & $1 / 2 \mathrm{SM} / \mathrm{mês}<\mathrm{NV}<2 \mathrm{SM} / \mathrm{mês} ;$ \\
E - Extrema Pobreza & $1 / 4 \mathrm{SM} / \mathrm{mês}<\mathrm{NV}<1 / 2 \mathrm{SM} / \mathrm{mês} ;$ \\
\hline
\end{tabular}

Fonte: ASPF (2017).

\footnotetext{
${ }^{7}$ Para este trabalho, considera-se o salário mínimo vigente no ano de 2017, sendo o valor de R\$934,00.
} 


\section{RESULTADOS E DISCUSSÕES}

Uma das características da agricultura familiar é a produção para a subsistência, proporcionando a reprodução social no meio rural. Contudo, as famílias além de produzirem para o autoconsumo também produzem excedentes, que são vendidos, gerando renda para adquirir aquilo que não se pode produzir.

Na Amazônia, em particular ao Acre, possui uma particularidade em relação às demais regiões brasileiras, uma vez que nessa região existe uma grande concentração fluvial, algumas localidades ainda com difícil acesso, além de a malha viária em alguns municípios não apresentarem uma infraestrutura adequada no interior do Estado. Todas essas condições implicam na dificuldade produtiva da produção familiar rural do Acre, especialmente para o escoamento da produção aos principais centros de comercialização, sendo um dos principais entraves para a geração de renda e o desenvolvimento das famílias no meio rural.

O Estado do Acre apresenta duas mesorregiões, o Vale do Acre - onde está localizado a capital Rio Branco -, que apresenta maior dinamismo por ter uma infraestrutura mais adequada, como o acesso dos municípios ocorrer por via fluvial e terrestre, ramais com condições de tráfego, energia elétrica disponível, e o Vale do Juruá, que apresenta ainda algumas dificuldades estruturais, como o isolamento terrestre de alguns municípios - Jordão, Santa Rosa do Purus, Marechal Thaumaturgo e Porto Walter -, ocorrendo apenas por via aérea e fluvial; energia elétrica deficitária, sendo adotado o sistema de rodízio de bairros em alguns municípios para a distribuição de energia etc.

Entretanto, mesmo com as dificuldades que o Estado do Acre ainda encontra para se desenvolver, a produção familiar rural revela-se um setor importante nesse processo de desenvolvimento rural. Conforme a Tabela 3, a composição da renda bruta do Vale do Acre, no período compreendido pelos anos agrícolas 1996/1997 a 2005/2006, foi marcado pelo fortalecimento dos produtos característicos dos sistemas extrativistas e agroflorestal, respectivamente, castanha do brasil e palmito de pupunha, bem como a consolidação da pecuária bovina como principal geradora de receita do sistema agrícola.

Já no Vale do Juruá, no período compreendido pelos anos agrícolas 1999/2001 a 2006/2007, a geração de renda nos sistemas produtivos está cada vez mais ligada à produção de macaxeira - notadamente seu principal subproduto, ou seja, a farinha de macaxeira -, sendo responsável por mais de $70 \%$ da renda gerada.

Tabela 2 - Evolução da Geração de Renda Bruta por Linha de Exploração, Vale do Acre, 2005/2006, e Vale do Juruá, 2006/2007, Acre - Brasil.

\begin{tabular}{|c|c|c|c|c|c|}
\hline \multirow{2}{*}{$\begin{array}{l}\text { Linha de } \\
\text { Exploração }\end{array}$} & Geração de & enda Bruta & \multirow{2}{*}{$\begin{array}{l}\text { Linhas de } \\
\text { Exploração }\end{array}$} & \multicolumn{2}{|c|}{ Geração de Renda Bruta (\%) } \\
\hline & \multicolumn{2}{|c|}{ Vale do Acre } & & \multicolumn{2}{|c|}{ Vale do Juruá } \\
\hline & $2005 / 2006$ & Evolução & & $2006 / 2007$ & Evolução \\
\hline Criações & 54,56 & 20,75 & Criações & 29,31 & 8,85 \\
\hline Criação de Bois & 47,48 & 46,43 & Criação de & 23,52 & 12,93 \\
\hline
\end{tabular}




\begin{tabular}{|c|c|c|c|c|c|}
\hline \multirow[b]{2}{*}{ Criação de } & \multicolumn{4}{|c|}{ TI } & \multirow[b]{2}{*}{$-{ }^{*}$} \\
\hline & 3,27 & $-59,94$ & $-{ }^{\star}$ & $-{ }^{*}$ & \\
\hline Criação de Aves & 2,73 & $-22,96$ & $-*$ & $-^{*}$ & $-{ }^{*}$ \\
\hline Outros & 1,08 & 2,27 & Outros & 5,79 & $-4,08$ \\
\hline Agricultura & 27,25 & $-34,27$ & Agricultura & 74,18 & 9,74 \\
\hline Café & 2,4 & 67,82 & $-*$ & $-^{*}$ & \\
\hline Macaxeira & 7,72 & 68,48 & Macaxeira & 57,68 & 18,92 \\
\hline Banana & 2,75 & $-36,29$ & $-{ }^{*}$ & $-{ }^{*}$ & $-{ }^{*}$ \\
\hline Melancia & 1,45 & $-30,87$ & $-{ }^{\star}$ & $-{ }^{*}$ & $-{ }^{*}$ \\
\hline Feijão & 1,19 & $-83,48$ & $-*$ & $-{ }^{*}$ & $-{ }^{*}$ \\
\hline Arroz & 2,03 & $-74,66$ & Arroz & 4,82 & $-8,34$ \\
\hline Milho & 2,46 & $-74,7$ & $-*$ & $-{ }^{*}$ & $-{ }^{*}$ \\
\hline Outros & 7,24 & 78,21 & Outros & 11,68 & $-0,84$ \\
\hline Agroflorestal & 5,62 & $-14,58$ & $-*$ & $-*$ & $-*$ \\
\hline Pupunha Fruto & 2,04 & $-35,33$ & $-*$ & $-{ }^{*}$ & $-*$ \\
\hline Cupuaçu & 1,21 & $-49,48$ & $-^{*}$ & $-{ }^{*}$ & $-^{*}$ \\
\hline $\begin{array}{l}\text { Pupunha- } \\
\text { Palmito }\end{array}$ & 0,76 & $5.149,71$ & $-{ }^{\star}$ & $-{ }^{*}$ & $-{ }^{*}$ \\
\hline Café & 1,13 & $-{ }^{\star}$ & $-\star$ & $-{ }^{*}$ & $-{ }^{*}$ \\
\hline Outros & 0,47 & $-52,96$ & $-^{*}$ & $-*$ & $-{ }^{*}$ \\
\hline Extrativismo & 12,58 & 85,47 & Extrativismo & 2,19 & $-12,91$ \\
\hline Castanha & 6,7 & 140,04 & $-{ }^{\star}$ & $-{ }^{*}$ & $-{ }^{*}$ \\
\hline Borracha & 1,97 & $-0,01$ & Borracha & 1,16 & $-5,71$ \\
\hline Madeira & 2,64 & 49,4 & Madeira & 1,03 & $-5,28$ \\
\hline Outros & 1,26 & 406,73 & Outros & $-{ }^{*}$ & $-{ }^{*}$ \\
\hline
\end{tabular}

Fonte: ASPF (2017).

Na composição da renda bruta das famílias do Vale do Acre destaca-se a diversificação produtiva nessa região. Observa-se que em todos os sistemas produtivos (criação, agricultura, agroflorestal e extrativismo) existe vários produtos que compõe a renda. Isso é uma característica fundamental para a agricultura familiar, tendo em vista as flutuações excessivas dos produtos agrícolas, assim, a diversificação da produção serve como uma precaução diante de quedas repentinas de preços em determinados produtos, que acarretam prejuízos as unidades produtivas familiares. Entretanto, as famílias produtoras rurais do Vale do Juruá não apresentam a mesma condição, sendo os principais produtos que compõem a renda oriundos da agricultura, que, geralmente, são pouco valorizados no mercado e para se ter um rendimento econômico considerável é necessário que se tenha escala de produção. Assim, nessa região as únicas atividades que demonstraram evolução foram a criação e a agricultura.

A criação de animais apresentou uma forte evolução na participação de renda bruta da produção familiar rural acreana, notadamente a criação de gado bovino. No Vale do Acre, esse aumento foi ainda maior, sendo a principal atividade geradora de renda com quase $50 \%$ de participação. É importante destacar que isso é uma situação preocupante e desperta atenção para que outras alternativas produtivas sejam incentivadas, uma vez que a criação de gado bovino apresenta problemas sociais, ambientais e econômicos para a produção familiar rural. Do ponto de vista econômico e social, trata-se de uma atividade altamente concentradora de renda e de terra, bem como sua 
viabilidade necessita tecnologia e investimentos adequados, o que nem sempre a agricultura familiar tem disponível. Do ponto de vista ambiental, são necessárias extensas áreas de terra para que seja uma atividade viável economicamente, o que acarreta no incentivo ao desmatamento na Amazônia. Assim, essa atividade é mais praticada como uma "reserva de valor" na produção familiar do Acre.

Por outro lado, em relação a produção de origem extrativa, observa-se que apesar ainda da ínfima participação na geração de renda bruta no Vale do Acre houve uma elevação considerável em relação ao período anterior. Esse aumento ocorreu, principalmente, pela valorização do preço da castanha do brasil no Estado do Acre nos últimos anos a partir da certificação ${ }^{8}$. Além disso, com a consolidação dos polos moveleiros em alguns municípios do Estado mediante incentivos do governo estadual, a atividade madeireira também mostrou um expressivo aumento. Já o Vale do Juruá mostra que as atividades extrativistas estão cada vez mais deixando de apresentar impacto na geração de renda para a produção familiar rural, talvez, em decorrência pela baixa incidência de usinas de beneficiamento de produtos dessa origem.

Assim, no Vale do Juruá, por um lado, a queda da produção da borracha e da castanha libera força de trabalho para outras atividades produtivas bem como para o assalariamento fora da unidade de produção familiar. Por outro lado, o aumento da produção de farinha provoca um aumento dos bens comprados no mercado (insumos para a nova linha de exploração) e um leve aumento dos custos fixos, tendo em vista que as unidades produtivas familiares já possuem a estrutura para a produção da farinha. Destaca-se que esse grande aumento constatado na produção de macaxeira ocorreu, principalmente, pelos incentivos do governo estadual com a construção de várias casas de farinhas com uma política para tentar viabilizar a produção familiar rural nessa região do Estado.

Da composição da receita dos sistemas de produção do Estado do Acre verifica-se que quando determinado sistema não possui produto característico, a criação de bois assume essa posição de forma potencializada. Observa-se que a criação de bois apresenta metade da geração de renda.

As dificuldades produtivas encontradas pela a agricultura familiar no Acre reflete-se no desempenho econômico. Observa-se na Tabela 4 uma situação de prejuízo, em termos medianos, entre as unidades produtivas familiares, cuja evolução denota ser um processo contínuo e substancial de redução de eficiência, observado por variação negativa do IEE de 56,65\% no Vale do Acre e $55,28 \%$ no Vale do Juruá.

Tabela 3 - Evolução dos indicadores econômicos das famílias rurais do Vale do Acre, 2005-2006 e Vale do Juruá, 2006-2007, Acre.

\begin{tabular}{cccccc}
\hline Indicadores & Unidade & $\begin{array}{c}\text { Vale do } \\
\text { Acre }\end{array}$ & $\begin{array}{c}\text { Evolução } \\
(\%)^{*}\end{array}$ & $\begin{array}{c}\text { Vale do } \\
\text { Juruá }\end{array}$ & Evolução (\%) $^{* *}$ \\
\hline $\mathrm{RB}$ & $\mathrm{R} \$ /$ mês & 915,85 & 12,55 & 452,07 & $-28,53$ \\
$\mathrm{RBT}$ & $\mathrm{R} \$ /$ mês & $1.227,70$ & 39,78 & 835,64 & 15,94 \\
$\mathrm{MBF}$ & $\mathrm{R} \$ /$ mês & 729,40 & 5,38 & 368,49 & $-14,74$ \\
$\mathrm{CF}$ & $\mathrm{R} \$ /$ mês & 605,48 & 180,66 & 663,56 & 112,97
\end{tabular}

${ }^{8}$ Ver Maciel (2007). 


\begin{tabular}{cccccc}
$\mathrm{CV}$ & $\mathrm{R} \$ / \mathrm{mês}$ & 202,38 & 18,34 & 183,88 & 11,11 \\
$\mathrm{VBCC}$ & $\mathrm{R} \$ /$ mês & 953,35 & 129,01 & $2.110,28$ & 544,17 \\
$\mathrm{LDM}$ & $\mathrm{R} \$ /$ mês & $1.757,58$ & 142,73 & $1.976,38$ & 180,7 \\
$\mathrm{AC}$ & $\mathrm{R} \$ /$ mês & 311,16 & $-70,47$ & 240,29 & $-66,65$ \\
$\mathrm{NV}$ & $\mathrm{R} \$ /$ mês & $1.142,46$ & $-43,8$ & 735,21 & $-43,19$ \\
$\mathrm{MBF} / \mathrm{Qh} / \mathrm{d}$ & $\mathrm{R} \$ /$ mês & 53,40 & 8,62 & 29,21 & 44,62 \\
$\mathrm{IEE}$ & und. & 0,94 & $-56,65$ & 0,55 & $-55,28$ \\
\hline
\end{tabular}

Obs.: Resultados medianos por UPF; ${ }^{*}$ Relativo ao período $1996 / 1997 ;{ }^{* *}$ Relativo ao período 1999/2001. Atualização monetária até abril de 2018 (INPC/IBGE).

Fonte: ASPF (2018).

De acordo com a Tabela 4, observa-se que a renda bruta gerada pelas famílias do Vale do Acre é mais que o dobro das famílias do Vale do Juruá. Esse desempenho superior dessas famílias está relacionado, principalmente, a diversificação produtiva, a maior facilidade de acesso aos mercados e oferta de produtos mais valorizados no mercado.

Entretanto, percebe-se que o baixo desempenho econômico da produção familiar rural acreana relaciona-se claramente no desequilíbrio de evolução entre a renda e custos produtivos, especialmente os fixos, que cresceram quase $200 \%$, enquanto que a renda auferida aumentou apenas $12,5 \%$, no Vale do Acre e no Vale do Juruá, os custos fixos aumentaram mais de $100 \%$ e a renda mostrou uma elevada redução de $28 \%$.

Dessa forma, os valores embolsados oriundos do processo produtivo (representados pela MBF) pelos produtores tornam-se insuficientes para os gastos realizados no mercado (conforme a LDM), dificultando-se a reprodução social das famílias. Destaca-se para o aumento substancial da dependência de mercado da produção familiar rural acreana, mostrando que nas duas regiões as famílias mais que dobraram esse indicador. Esse aumento expressivo da linha de dependência do mercado demanda atividades produtivas eficientes economicamente, e, portanto, geradoras de receita capaz de fazer face às necessidades da unidade produtiva e das famílias.

Não obstante, destaca-se o papel das transferências governamentais, além do assalariamento fora da unidade produtiva familiar, para aumentar a renda disponível (ver RBT) aos produtores a fim de fazer frente aos gastos no mercado; contudo, ainda insuficiente, já que, confrontando a RBT e a LDM, ficam descobertos os gastos necessários no mercado das famílias do Vale do Acre em torno de $30 \%$. Já no Vale do Juruá, a situação é ainda mais complicada, apresentando $60 \%$ de gastos superiores a renda auferida. Isso indica uma situação clara de endividamento por parte das famílias, refletindo-se como alternativa para garantir sua manutenção a venda de bens, levando a perda de patrimônio; comprar fiado; pegar dinheiro emprestado; e, em último caso, a opção é vender a propriedade para quitar as dívidas.

Outro aspecto preocupante nos resultados é a piora no nível de vida das famílias rurais do Estado do Acre, uma vez que está associada com a forte redução (cerca de $70 \%$ ) da produção para o autoconsumo, aumentando, por outro lado, a dependência do mercado.

De modo geral, há uma redução tanto no nível de vida quanto no autoconsumo das famílias rurais acreanas, comparando-se com o primeiro período. $\mathrm{O}$ autoconsumo $(\mathrm{AC})$ é o indicador com maior redução no período 
analisado e essa redução reflete na maior parte dos outros indicadores apresentados na Tabela 4, como o VBCC, pois na medida em que as famílias deixam de produzir para o autoconsumo, elas passam a comprar os alimentos no mercado. Outro indicador que sofre alteração por causa da redução do AC é o nível de vida, que também sofreu uma grande redução no período analisado. Essa redução do nível de vida das famílias rurais do Estado do Acre reflete no aumento da pobreza, significando que o poder aquisitivo das famílias diminuiu ao longo do período. O autoconsumo é uma das características da agricultura familiar, bem como uma de suas fortalezas para a reprodução social e desenvolvimento no campo, por isso, sua redução torna-se uma grande preocupação.

Agora, deve-se ressaltar que, mesmo com as dificuldades de reprodução das famílias do Vale do Acre, ainda compensa insistir em produzir no meio rural dessa região, uma vez que o nível de vida em termos monetários supera em um pouco mais que a metade do salário mínimo vigente no país. Além disso, a remuneração da mão de obra familiar $(\mathrm{MBF} / \mathrm{Qh} / \mathrm{d})$ é maior que o valor do custo de oportunidade da região ( $R \$ 50,00)$, no período considerado. Entretanto, essa condição não se estende para as famílias rurais do Vale do Juruá, uma vez que apresenta um nível de vida inferior (aproximadamente $20 \%$ ) ao salário mínimo.

Do ponto de vista de distribuição de renda, de acordo com a Tabela 5 as unidades produtivas familiares do Estado do Acre segue o mesmo padrão concentrador de renda constatado no processo de desenvolvimento brasileiro ao longo do tempo.

Contudo, mesmo com uma alta concentração de renda na região do Vale do Acre, nota-se uma redução da desigualdade de renda em torno de $14 \%$ para um período em torno de uma década e para a região do Vale do Juruá constatou-se uma redução de $6 \%$.

Tabela 4 - Evolução dos indicadores de desigualdade de renda, Vale do Acre, 2005/2006, e Vale do Juruá, 2006/2001, Acre - Brasil.

\begin{tabular}{lcccc}
\hline Descrição & $\begin{array}{c}\text { Vale do } \\
\text { Acre }\end{array}$ & Evolução (\%)* & $\begin{array}{c}\text { Vale do } \\
\text { Juruá }\end{array}$ & $\begin{array}{c}\text { Evolução } \\
(\%)^{\star *}\end{array}$ \\
\hline Índice de Gini & 0,51 & $-14,42$ & 0,49 & $-6,6$ \\
$\%$ renda 10+ & $34 \%$ & 48,82 & $29 \%$ & 10,37 \\
$\%$ renda 50- & $27 \%$ & 74,56 & $32 \%$ & $-4,29$ \\
10\% rico / 50\% & 1,24 & $-14,74$ & 0,89 & 15,32 \\
pobre & &
\end{tabular}

Obs.: *Relativo ao período 1996/1997; **Relativo ao período 1999/2001.

Fonte: ASPF (2017).

Cabe ressaltar que a melhoria na distribuição de renda entre os produtores familiares rurais do Estado do Acre ocorreu, principalmente, nos sistemas extrativistas, denotando o importante papel desenhado pelos movimentos sociais, na busca de políticas públicas mais igualitárias e adequadas, destacando-se, por um lado, a criação da Lei Chico Mendes ${ }^{9}$ que

\footnotetext{
${ }^{9}$ Lei estadual $n^{\circ} 1.277 / 99$, em vigor no Estado do Acre, que paga atualmente aos produtores de borracha o valor de $\mathrm{R} \$ 1,40 / \mathrm{kg}$ de borracha como forma de pagamento por serviços ambientais.
} 
incentivou as famílias das Reservas Extrativistas (RESEX) e, por outro, o cooperativismo entre os produtores.

Ademais, observa-se a efetividade dessa mudança de distribuição de renda na diferença entre os $10 \%$ mais "ricos" e os $50 \%$ mais "pobres" no Vale do Acre, uma vez que a queda da desigualdade beneficiou a população mais pobre na mesma proporção. Porém, observa-se no Vale do Juruá, essa diferença se ampliou. Assim, esse movimento pode ser mais bem analisado mediante a delimitação da linha de pobreza entre as famílias da região estudada.

Vale destacar que a melhoria no índice de Gini não significa que houve um desenvolvimento no bem estar da população. Apenas aponta que a situação da distribuição da renda na região é mais igualitária, mesmo se a renda for mais baixa que outras regiões, uma vez que esse índice mensura apenas o nível de concentração de renda e não a qualidade de vida ou nível de pobreza.

Para tanto, o nível de vida (NV) é o valor total apropriado pelo produtor familiar, em termos monetários. Por isso, esse indicador é o mais adequado para mensurar a condição da produção familiar rural, comparando-o com o salário mínimo vigente no período avaliado.

A Tabela 6 apresenta uma estratificação das famílias do Vale do Acre e Vale do Juruá, de acordo com o NV, buscando mensurar o nível de pobreza das famílias rurais no Estado do Acre.

Tabela 5 - Percentual de Famílias por estrato do Nível de Vida, em termos monetários, do Vale do Acre 1996-1997/2005-2006 e Vale do Juruá 1999-2001/20062007

\begin{tabular}{ccccc}
\hline Estratos & $\begin{array}{c}\text { Vale do Acre } \\
\mathbf{2 0 0 5 / 2 0 0 6}\end{array}$ & Evolução (\%) $^{\star}$ & $\begin{array}{c}\text { Vale do Juruá } \\
\mathbf{2 0 0 6 / 2 0 0 7}\end{array}$ & Evolução (\%) $^{\star \star}$ \\
\hline A & $9 \%$ & $-74 \%$ & $1 \%$ & $-88 \%$ \\
B & $18 \%$ & $-35 \%$ & $11 \%$ & $-63 \%$ \\
C (a) & $39 \%$ & $812 \%$ & $30 \%$ & $-25 \%$ \\
C (b) & $21 \%$ & $-38 \%$ & $31 \%$ & $163 \%$ \\
D & $10 \%$ & $577 \%$ & $17 \%$ & $233 \%$ \\
E & $5 \%$ & $552 \%$ & $10 \%$ & $394 \%$ \\
\hline
\end{tabular}

Obs.: *Relativo ao período 1996/1997; ${ }^{* \star}$ Relativo ao período 1999/2001.

Fonte: ASPF (2017).

De modo geral, nota-se que $10 \%$ das famílias rurais do Vale do Acre, no período analisado, encontram-se em situação de pobreza e 5\% encontram-se em situação de extrema pobreza. No Vale do Juruá a situação é um pouco pior, com $17 \%$ das famílias rurais em situação de pobreza e $10 \%$ em situação de extrema pobreza. Destarte, destaca-se que o número de pobres (estrato D) e indigentes (estrato $\mathrm{E}$ ) cresceu em números preocupantes no período analisado, com um aumento na quantidade de pobres em 577\% e de indigentes em $552 \%$ no Vale do Acre. No Vale do Juruá, o crescimento foi de $223 \%$ no número de pobres e $394 \%$ de indigentes.

A partir da Tabela 6 é possível também definir o percentual de famílias em estado de insegurança alimentar, pois de acordo com o procedimento da Pesquisa Nacional por Amostra de Domicílio (PNAD) 2004, realizado pelo Instituto Brasileiro de Geografia e Estatística (IBGE), as famílias que vivem com 
renda per capita/mês abaixo de $1 / 4$ salário mínimo estão em situação de insegurança alimentar, desta forma, pode-se dizer que as famílias do estrato $\mathrm{E}$ estão com insegurança alimentar, ou seja, 5\% das famílias rurais do Vale do Acre e $10 \%$ do Vale do Juruá apresentam insegurança alimentar no período analisado.

Obviamente que as causas desses resultados estão também relacionadas aos baixíssimos rendimentos auferidos no processo produtivo pelas famílias, talvez como consequência dos baixos níveis de autoconsumo, levando ao círculo vicioso que movimentou negativamente as famílias dos extratos superiores (A, B e $C$ ) aos inferiores.

O aumento do número de famílias em situação de pobreza e extrema pobreza, assim como a presença de insegurança alimentar nas famílias rurais do Estado do Acre, ocasionado principalmente pela redução da produção para autoconsumo, gera um grande impacto no que diz respeito à reprodução social dessas famílias, pois defronte desses problemas muitas famílias abandonam sua propriedade rural em busca de melhores condições de vida, o que acaba na maioria das vezes sendo uma alternativa inviável, já que geralmente possuem baixo nível de escolaridade e quase nenhuma qualificação profissional, assim, dificilmente encontrarão oportunidade de emprego na cidade.

\section{CONSIDERAÇÕES FINAIS}

Nos últimos anos o conceito de segurança alimentar vem ganhando espaço na elaboração de políticas públicas. É consenso da maioria dos autores que a questão da fome não se justifica por falta de alimentos, sendo considerada a pobreza como a primeira causa da fome e da desnutrição. Outro problema identificado está relacionado à insuficiência da produção de alimentos, além da má distribuição, dificultando o acesso da população a uma alimentação adequada.

O presente trabalho buscou identificar como a relação entre os níveis de pobreza e autoconsumo afetam a segurança alimentar da produção familiar rural do Estado do Acre, bem como identificar a distribuição de renda entre os produtores.

Dessa forma, observou-se que em geral a produção familiar rural acreana apresentou uma redução expressiva na sua viabilidade e eficiência econômica. Essa mudança ocorreu, notadamente, pelo desequilíbrio entre a evolução da receita auferida e do custo - especialmente, o custo fixo - das famílias.

Identificou-se que os produtores familiares rurais apresentaram uma mudança na estrutura produtiva no que diz respeito a geração de renda. Antes os produtos da agricultura apresentavam maior impacto na renda da produção familiar rural, no período que decorreu de quase dez anos, a criação de gado bovino mostrou-se como a produção com maior participação na geração de renda, tanto para as famílias do Vale do Acre como do Vale do Juruá.

Por outro lado, devido à valorização que alguns produtos de origem extrativista tiveram, a organização social em algumas localidades e a consolidação do mercado, como o caso da castanha e da madeira, essa produção mostrou uma evolução substancial na participação da geração de 
renda das famílias do Vale do Acre. Entretanto, no Vale do Juruá observou-se que houve uma redução considerável desses produtos, assim, as atividades migraram para a agricultura mediante produtos pouco valorizados no mercado - especialmente, a macaxeira e a farinha.

Do ponto de vista do autoconsumo, verificou-se uma forte redução nesse indicador para os produtores familiares rurais do Estado do Acre. Esse indicador é fundamental para a reprodução social das famílias no campo, uma vez que reduz consideravelmente os gastos no mercado, especialmente, a aquisição de alimentos. A redução desse indicador refletiu em uma maior dependência do mercado, que foi superior até a renda bruta total auferida pelas famílias, demonstrando sinais claros de endividamento das famílias.

Além disso, a redução do autoconsumo no caso da produção familiar rural afeta também o nível de vida. Observou-se que houve uma redução considerável nesse indicador da produção familiar rural acreana, mostrando que o padrão de vida das famílias piorou em relação ao período anterior.

Do ponto de vista de distribuição de renda, verificou-se uma leve redução no índice de Gini dos produtores familiares rurais do Acre. Entretanto, a desigualdade de renda no Estado mostrou-se um acompanhamento dos padrões brasileiros, especialmente, pela mudança produtiva que ocorreu ao potencializar a criação de gado bovino em substituição as atividades agrícolas, agroflorestais e extrativistas.

A distribuição de renda de forma desequilibrada e o baixo nível de autoconsumo se refletiu no número de famílias em situação de pobreza e extrema pobreza, ou seja, em situação de insegurança alimentar. Contatou-se que houve uma redução no número de famílias que estavam em uma faixa considerada boa (estratos A, B e C) para os padrões rurais e um aumento excessivo no número de famílias pobres e indigentes. Portanto, não houve uma distribuição de renda, mas uma distribuição da pobreza de forma mais igualitária.

Observou-se que o autoconsumo gerou uma espécie de "efeito dominó", uma vez que a produção familiar rural aumentou os gastos no mercado; reduziu o nível de vida, aumentando os níveis de pobreza e piorando a distribuição de renda. Assim, os problemas encontrados no presente trabalho podem ser encaminhados mediante o incentivo ao autoconsumo. Claro que a viabilidade da produção familiar rural do Acre não depende exclusivamente disso, mas não se pode negligenciar o papel fundamental que esse indicador representa para as famílias.

Além disso, a implantação de políticas públicas que voltem a incentivar alternativas produtivas, como as atividades agrícolas, agroflorestais e extrativistas são fundamentais para viabilizar a produção familiar rural acreana de modo que tirem o foco da atividade pecuária, uma vez que trata-se de uma atividade contrária aos padrões de desenvolvimento da agricultura familiar e, principalmente, da região Amazônica.

\section{REFERENCIAL BIBLIOGRÁFICO}

ASPF - Análise Socioeconômica de Sistemas Básicos de Produção
Familiar Rural no Estado Acre. 2018. Disponível em: 
$<$ http://aspf.wordpress.com>. (Projeto de Pesquisa do Centro de Ciências Jurídicas e Sociais Aplicadas da UFAC).

BARROS, R. P. de; et. al: A estabilidade inaceitável: desigualdade e pobreza no Brasil. Texto para discussão nำ 800, IPEA 2001.

CACCIAMALI, Maria Cristina. Distribuição de renda no Brasil: Persistência do elevado grau de desigualdade. In: Manual de Economia, São Paulo: Ed. Saraiva, 2002, p. 406-422.

CAMARANO, A. A.; ABRAMOVAY, R. Éxodo rural, envelhecimento e masculinização no Brasil: panorama dos últimos 50 anos. Rio de Janeiro: IPEA, $1999 . \quad 28 p . \quad$ Disponível em: <http://www.dominiopublico.gov.br/download/texto/td_0621.pdf>. Acesso em: 13 nov. 2012.

CHAYANOV, A. V. La organisación de la unidad económica campesina. Buenos Aires, Nueva Vision, 1974.

DEVES DIEL OTÁVIO; FILIPPI E. E.: A Segurança Alimentar e as experiências das Políticas Agro-Alimentares Locais no Fortalecimento da Agricultura Familiar. In. IV Congresso Internacional de la RedSial , 2008, Mar del Plata Argentina.

DOMBEK, Luiz Antônio. Autoconsumo e Segurança Alimentar em Assentamentos Rurais do Portal do Panorama. Campinas: UNICAMP/FEA, 2006. 220p. (Dissertação de mestrado).

GAZOLLA, Marcio; SCHNEIDER Sergio. A PRODUÇÃO DA AUTONOMIA: os "papéis" do autoconsumo na reprodução social dos agricultores familiares. Artigo publicado na Revista Estudos Sociedade e Agricultura, Rio de Janeiro, (UFRRJ), v15, p. 89-122, 2007.

GUANZIROLI, Carlos et al. Agricultura Familiar e Reforma Agrária no Século XXI. Rio de Janeiro: Garamond, 2001.

HOFFMANN, Rodolfo; Desigualdade da distribuição da renda no Brasil: a contribuição de aposentadorias e pensões e de outras parcelas do rendimento domiciliar per capita. Artigo publicado na Revista Economia e Sociedade, Campinas, v. 18, n. 1 (35), p. 213-231, abr. 2009.

HOFFMANN, Rodolfo. Pobreza, insegurança alimentar e desnutrição no Brasil. Estudos Avançados vol. 9 no. 24 São Paulo May/Aug. 1995. Acesso em 09/06/2014. http:// www.scielo.br/scielo.php?pid=S0103$\underline{40141995000200007 \& \text { script }=\text { sciarttext. }}$.

IBGE, Pesquisa Nacional por Amostra de Domicílios. Rio de Janeiro de 2010. 
INSTITUTO DE PESQUISA ECONÔMICA APLICADA. Resultados GINI. Brasília, 2017. Disponível em: http://www.ipeadata.gov.br/. Acesso em 02 de janeiro 2017.

MACIEL, R. C. G. Certificação Ambiental: uma estratégia para a conservação da floresta amazônica. Campinas: [s.n.], 2007. 175 p. (Tese de Doutorado Economia Aplicada, IE/UNICAMP). Disponível em: http://libdigi.unicamp.br/document/?code=vtls000417323

MALUF, Renato S. Segurança Alimentar e Nutricional. Editora Vozes, 3a Edição, 2007.

.; MENEZES, Francisco; MARQUES, B. Susana. Caderno

"Segurança Alimentar". Disponível em:

http://www.forumsocialmundial.org.br/download/tconferencias Maluf Menezes 2000 por.pdf. Acesso em 22/01/2013

MATTE, A; SPANEVELLO, R.M; AZEVEDO, L.F de. A reprodução social na agricultura familiar: A saída dos filhos e o encaminhamento do patrimônio entre agricultores sem sucessores. In: Congresso Brasileiro de Economia, Administração e Sociologia Rural, XLVIII, 2010, Campo Grande. Anais... Campo Grande: SOBER, 2010.

NAVARRO, Zander. Desenvolvimento rural no Brasil: os limites do passado e os caminhos do futuro. Estudos Avançados, São Paulo, v. 15, n. 43, p. 83100, set./dez. 2001.

NEDER, Dantas Henrique. Um estudo sobre a pobreza rural com abordagem multivariada. In. Congresso da Sociedade Brasileira de Economia, Administração e Sociologia Rural, 2008, Rio Branco: SOBER, 2008.

OLIVEIRA, Régis Borges de: Conceitos e principais métodos Existentes para mensuração da Pobreza no brasil. IICA, Campinas, 30 de junho de 2010

OLALDE, A. R. ; PORTUGAL, Cadja Araújo . Agricultura Familiar, Reforma Agrária e sua inserção no enfoque territorial no Brasil. . In: XLII Congresso Brasieliro de Economia e Sociologia Rural, 2004, Cuiabá. XLII Congresso Brasileiro de Economia e Sociologia Rural, 2004.

PELEGRINI Gelson; GAZOLLA Marcio; A agroindustrialização da produção como estratégia de reprodução social da agricultura familiar. Artigo publicado na Revista Estudos Sociais e Agrícola, Rio de Janeiro, v17, N2 p. 323-378. 2009.

ROCHA, Sonia; Pobreza e indigência no Brasil - algumas evidências empíricas com base na PNAD 2004. Artigo publicado na Revista nova Economia Belo Horizonte 16 (2) 265-299_maio-agosto de 2006. 
SCHNEIDER, Sérgio. Teoria social, agricultura Familiar e pluriatividade. Artigo publicado na Revista Brasileira de Ciências Sociais, v18, N51 p. 100121, FEV. 2003.

SILVA, A. M. R. Um estudo sobre pobreza multidimensional na região Nordeste do Brasil. Uberlandia: UFU, 2009. (Dissertação de mestrado).

SOUZA FILHO, H. M.; BUAINAIN, A. M., SILVEIRA, J.M.F.; VINHOLIS, M. M. B. Condicionantes da adoção de inovações tecnológicas na agricultura. Cadernos de Ciência \& Tecnologia, Brasília, v. 28, n. 1, p. 223-255, jan./abr. 2011.

SPANEVELLO, Marisa R.; LAGO Adriano. A transmissão do patrimônio na agricultura familiar: uma análise a partir da compensação das filhas. Artigo publicado na Revista Internacional Interdisciplinar INTERTHESIS, Florianópolis - SC - Brasil, v7, N1 p. 222-141, JAN/JUN. 2010.

TURPIN, M. Elena. A Alimentação Escolar como Fator de Desenvolvimento Local por meio do Apoio aos Agricultores Familiares. Campinas 2009. Disponível em http://www.unicamp.br/nepa/arquivo san/volume 162 2009/2 Marina MariaE lenaTurpin\%5B20-42\%5D.pdf. Acesso em 17 de janeiro de 2013.

VEIGA, José Eli da. O Brasil rural ainda não encontrou seu eixo de desenvolvimento. Estudos Avançados 15 (43), 2001.

WANDERLEY, M. de N. B., Agricultura familiar e campesinato: rupturas e continuidade, Estudos Sociedade e Agricultura, Rio de Janeiro, n. 21, p. 4261 , out. 2003.

Raízes Históricas do Campesinato Brasileiro, Minas Gerais: XX Encontro Anual da ANPOS. GT17. Processos Sociais Agrários, out. 1996.

WOLF, Eric R. Sociedades Camponesas. Rio de Janeiro, 1970.

WORLD BANK. Poverty and Shared Prosperity 2016: Taking on Inequality" Washington, DC: World Bank; New York: Oxford University Press. 2016. 193p. 